\title{
Transition phase of connectivity for wireless networks with growing process
}

\section{Bo Gu and Xiaoyan Hong*}

\author{
Department of Computer Science, \\ University of Alabama, \\ Tuscaloosa, AL 35487, USA \\ E-mail: bgu@cs.ua.edu \\ E-mail: hxy@cs.ua.edu \\ *Corresponding author
}

\begin{abstract}
The connectivity of wireless network is critical for effective and efficient communications. Yet, it is challenged by mobility, transmission range and density. In terms of increasing node density, there is a transition phase of connectivity when a network starts transforming from partition to connected state. In this paper, we study the upper- and lowerbounds of critical density for the transition phase using percolation theory. We further derive critical time points for the bounds according to three growth functions of node density. The simulation demonstrates the evolution of network connectivity and validates the connectivity transition times.
\end{abstract}

Keywords: wireless network; connectivity; Percolation theory.

Reference to this paper should be made as follows: Gu, B. and Hang, X. (2012) 'Transition phase of connectivity for wireless networks with growing process', Int. J. Ad Hoc and Ubiquitous Computing, Vol. 11, Nos. 2/3, pp.169-177.

Biographical notes: $\mathrm{Bo} \mathrm{Gu}$ is a $\mathrm{PhD}$ Student in the Department of Computer Science at the University of Alabama. He received MS Degree in Computer Science from the University of Alabama at 2009 and MS Degree in Computer Science from Beihang University, China at 2007. His research interests include wireless networking, mobile networking, delay tolerant network and network modelling.

Xiaoyan Hong is an Associate Professor in the Computer Science Department at the University of Alabama. She completed her PhD Degree from the University of California, Los Angeles in 2003. Her research interests include wireless networks, mobile computing and wireless sensor networks.

\section{Introduction}

In a wireless network, the connectivity in multi-hop way is often affected by node density, transmission range, residual energy and node mobility. A partitioned wireless ad hoc network can turn into a connected network when some of the above factors change. The number of nodes being deployed in a designated area can influence the network connectivity greatly. Understanding the transition phase of the network between partition and connectivity can help network management, planning, maintenance and performance monitoring. The importance of the connectivity issue has drawn great attention recently to obtain fundamental properties of the problem in many application domains. For example, the minimum number of average neighbours for network connectivity in static topology (Xue and Kumar, 2004); the last connection time and the first partition time with regard to node failure models in wireless networks (Xing and Wang, 2008); the impact of interference on the connectivity (Dousse et al., 2005); the relationship between power saving on sensor networks and maintaining connectivity (Dousse et al., 2004; Kong and Yeh, 2007). In these works, the percolation theory has been used leading to results of critical densities defined based on statistical concept of giant component (Kesten, 1982).

Viewing the need of parsimonious use of networking devices in some applications, for example, a large area with scarce available resources like in some surveillance scenarios, an understanding of the transition phase becomes extremely important. The transition phase can be defined by the lower and the upper bounds of the critical density following the growth of number of nodes. In the early related works, the problem has been simplified for uniformed networks which are not sufficient for some heterogeneous and multi-hop wireless network scenarios. In heterogeneous and multihop wireless networks, nodes bearing differences in their capability of communications, e.g., the transmission power, the residual energy at wireless nodes, will lead to various transmission ranges. And further, nodes equipped with adjustable antennas may have 
adaptable transmission ranges. In this paper, we revise the analytic modeling procedure to reflect the need for a variable transmission range $r$ and the multi-hop forwarding using the percolation theory in deriving the two bounds.

The transition phase of the wireless network connectivity can be illustrated according to the growth of node density in the following way. In the beginning, a small amount of wireless nodes are uniformly distributed in the area and the low density causes the network to be partitioned. By increasing the number of nodes, the network becomes dense and turns into a connected phase when the density exceeds a critical value. The evolution of connectivity is helpful for understanding the states of a network and leads to different applications corresponding to the states.

In this paper, we first derive the two bounds of the critical density of wireless ad hoc networks based on the percolation theory. Here, the lower bound defines the moment (called the first connection time) before which the network is partitioned because of the sparse density and after which the nodes in network gradually become connected in the same component. The upper bound defines the moment when all the nodes are connected and become the giant component with high probability (called total connection time). Then, we study the duration of the transition phase, i.e., the latency to reach the total connection starting from the first connection based on percolation theory. This latency highly depends on the potential growth process of the node density. Here we offer three growth functions to derive the first connection time and the total connection time which denote the points when the node densities reach the lower and upper bound of critical value respectively.

The paper is organised as follows. Section 2 summarises the related works that use the percolation theory for connectivity issue. In Section 3, we introduce the network model and preliminary knowledge related to the percolation that we use in this paper. The main analytical modeling of the bounds and results are given in Section 4. We also perform simulations and show case the connectivity based on the three growth functions and the related bounds in Section 5. The conclusion is presented in Section 6.

\section{Related work}

Percolation theory is widely used to analyse the network connectivity under various applications in recent publications. In Dousse et al. (2004), the power saving mechanism with completely uncoordinated pattern is discussed using continuum percolation theory. The bound of message latency, from a sender to its receiver, is derived by using an extension of the first passage percolation theory (Liggett, 1985). The authors in Kong and Yeh (2007) propose a distributed energy management algorithm for wireless sensor networks. By using the node degree and the percolation condition, the mechanism for saving energy can guarantee the network to be in connected state all the time. In addition, the work shows that the message delay scales sub-linearly or linearly with Euclidean distance between the source and the destination depending on network phase in terms of connectivity.
In Xing and Wang (2008), the percolation-theory based connectivity issue is discussed in order to derive the last connection time and the first partition time for wireless networks, where the failure nodes may cause the network to enter into a partitioned phase. In their analytic model, the network is mapped to a lattice and the network properties are converted to the corresponding features in the lattice. Then, the condition of connectivity in wireless network is obtained from percolation condition in lattice. Our work is relevant to the paper (Xing and Wang, 2008), but the number of wireless node in network grows as time goes, which is the opposite to the case in Xing and Wang (2008). In addition, a different method is employed to derive the critical density and related time points.

The critical transmission range for connectivity of wireless network is studied in Gupta and Kumar (1998). By deriving the lower and upper bounds of the probability for the connected network, the conclusion is that the network graph with $\pi r^{2}(n)=\frac{\log n+c(n)}{n}$ is connected with probability one as $n \rightarrow \infty$ if and only if $c(n) \rightarrow+\infty$. In Ramanathan and Rosales-hain (2000), the problem of transmission power adjustment for topology control is proposed and the objective is to minimise the maximum transmit power subjecting to connectivity constraint. In addition, the distributed algorithms are introduced for mobile network so as to adapt to the dynamic topology change. The topology control depending on the alteration of transmission range is also discussed in Sanchez et al. (1999) where the so called Direct neighbour Graph is generated first and the optimal range is derived based on Minimum Spanning Tree. Based on the geometric random graph and spatial analysis of wireless node, Bettstetter (2002) provides an analytical result to obtain the minimum node degree for $k$-connectivity network. A continuum framework of wireless and mobile network (Chen et al., 2011) is proposed to identify the connectivity status of network. The continuum space consists of two dimensions: node density and node speed. According to different combination of density and speed, three classes of network are found including the total connected network, disrupted network and partitioned network.

In this paper, we follow an alternative approach based on the technique mentioned in Meester and Roy (1996) to study the relation of node density and transmission range in terms of connectivity. And we consider the increment of node density, a different network scenario from the previous works. Furthermore, given the current node density, the density model can help to find the approximate critical transmission range for network connection.

\section{Problem modelling}

\subsection{Network model}

The network is described as the Poisson Boolean Model in two dimensions (Meester and Roy, 1996). It is introduced as follows. $\mathcal{B}(X, r, \lambda)$ is a union of randomly scattered disks:

$$
\left.\mathcal{B}(X, r, \lambda) \doteq \cup B\left(x_{i}, r_{i},\right) \lambda\right)
$$


where $B\left(x_{i}, r_{i}, \lambda\right)$ is a disk centered at $x_{i}$ having radius $r_{i} . x_{i}$ denotes the point of the Poisson process $X$ of density $\lambda$ in two dimensions. In order to model the wireless network, $x_{i}$ can be viewed as the wireless node $i$ and $r_{i}$ denotes its transmission range. For simplicity, we assume the transmission ranges of node are equal and denoted by $r$, so the connective condition for any two nodes is $\left\|x_{i}-x_{j}\right\| \leq r$.

\subsection{Percolation theory}

Percolation theory (Kesten, 1982; Bollobas and Riordan, 2006; Penrose, 2006) is initially used to model the spread of fluid or gas through a random medium. The original problem is that: Suppose a large porous rock is submerged under water for a long time, will the water reach the center of the stone? The theory assumes the surface is covered by points and points could be connected by edges. Given a large two dimensional grid of edges, an edge can be open with probability $p$ and closed with probability $q=1-p$.

The collection $W_{0}$ of points connected to source 0 by an open edge is called open cluster of source 0 . Most questions in percolation theory concern some aspects of the distribution of $W_{0}$. Let $P_{p}$ denote the probability on the configuration of open edge with probability $p$, and let $\theta(p)$ be the percolation probability defined as:

$$
\theta(p)=P_{p}\left\{\left|W_{0}\right|=\infty\right\}
$$

where $\left|W_{0}\right|$ is the number of points in $W_{0}$. In general, $\theta(p)$ is proportional to the probability $p$, and the following properties hold:

$$
\theta(0)=0, \quad \theta(1)=1 \text {. }
$$

And $p_{c}:=\sup \{p: \theta(p)=0\}$ is called critical probability

The percolation problem in geometric structure has been studied extensively in last three decades (Meester and Roy, 1996). However, when the position of node is expressed in a continuous space, the continuum model is responsible to describe the process of percolation. A fundamental result of continuum percolation is that there exists a critical density $\lambda_{c}$, defined by $\lambda_{c}=\inf \left\{\lambda>0: P_{\lambda}\left\{\left|W_{0}\right|=\infty\right\}>0\right\}$, so that: if $\lambda>\lambda_{c}$, the network is in the super-critical phase; while if $\lambda<\lambda_{c}$, the network is said to be sub-critical and $P_{\lambda}\left\{\left|W_{0}\right|=\infty\right\}=0$. When the graph stays in super-critical phase, $W_{0}$ is normally called the giant component since it would contain infinite number of nodes in network.

\subsection{Connectivity latency}

In order to model the transit phase from partition to connected state, we assume that the network initially contains only a small number of nodes expressed by the density $\lambda_{0}$ within a fixed network area. After that, we introduce the growth function of network denoted by $x(t)$ which describes the increment of the number of nodes against time. Then the density function is defined as follows according to the Thinning theorem (Penrose, 2006):

$$
\lambda(t)=\frac{\lambda_{0} x(t)}{x(0)}
$$

where $x(0)$ represents the initial number of nodes in the network. When the density increases, two time-based metrics are critical to understand the critical density. One metric is the first connection time $T_{f}$ and the other is the total connection time $T_{t}$. They are defined in equation (1) and equation (2) respectively.

$$
\begin{aligned}
& T_{f}=\sup \left\{t>0: P_{\lambda_{t}}\left\{\left|W_{0}\right|=\infty\right\}=0\right\} \\
& T_{t}=\inf \left\{t>0: P_{\lambda_{t}}\left\{\left|W_{0}\right|=\infty\right\}>0\right\} .
\end{aligned}
$$

Typically, the metric $T_{f}$ defines the latest time point in a density growing process before which the probability $P_{\lambda_{t}}$ is zero, i.e., the network is partitioned in terms of the giant component. The metric $T_{t}$ depicts the earliest time point in a density growth after which the probability becomes larger than zero, i.e., the network is connected in terms of the giant component. Thus, the two metrics define the time instances according to the lower bound and the upper bound of the critical density. Given the time function of node density and the range of critical density, we can derive these two time points.

\section{Analysis and result}

In this section, we first derive the lower bound and upper bound of $\lambda_{c}$. The variable of transmission range $r$ is considered in the function of density bound for studying the trend as the range varies. Secondly, the first connection time and total connection time are derived. Specifically, the sparse network with low density of node at time $t_{0}$ grows with certain distribution until the density reaches the lower bound of critical density at $t_{1}$. Then the first connection time will be $t_{1}$. After that, when the density increases to the upper bound at $t_{2}$, then $t_{2}$ becomes the total connection time.

\subsection{Lower bound of $\lambda_{c}$}

We derive the lower bound following the approach used in Meester and Roy (1996) by using Branching Process (Grimmett and Stirzaker, 1992). The rationale behind the approach is that we first derive the function about the number of nodes in the connected component and then find the minimum density requirement for a percolated network where the probability of having infinite number of node is greater than zero. We apply a two dimensional Poisson Boolean model $(X, r, \lambda)$ and a multi-type branching process where the type of node denotes the distance between a node and its predecessor. The distances are distributed over all real numbers in $(0, r)$ and the maximum value $r$ guarantees the link between the nodes in adjacent generation

Generally, the members of the $k$ th generation of the branching process starting from $x_{0}$ are $x_{k, 1}, x_{k, 2}, \ldots, x_{k, n_{k}}$. the 
children of $x_{k, i}, i \in\left[1, n_{k}\right]$ are those points from another independent process $X_{k+1}$ which fall in the region $S\left(x_{k, i}, r\right) \backslash S\left(x_{k-1, j}, r\right)$, where $x_{k-1, j}$ is the parent of $x_{k, i}$ and circle region $S(x, r)=\{y:|y-x| \leq r\}$, also the type of node $x_{k+1, l}$ at the $(\mathrm{k}+1)$ th generation from the process $x_{k+1}$ is $t:=\left|x_{k, i}-x_{k+1, l}\right| \in(0, r)$, in consequence, the distribution of node $x_{k+1, l}$ whose types lie in $(0, r)$ depends on the region $R$ :

$$
R=\left(S\left(x_{k, i}, r\right) \backslash S\left(x_{k-1, j}, r\right)\right) \cap\left\{y:\left|y-x_{k, i}\right| \in(0, r)\right\} .
$$

Assume that $x_{k, i}$ is of type $u$ and $x_{k+1, l}$ has type $v$, then the region $R$ becomes a curve defined as $\left(S\left(x_{k, i}, r\right) \backslash S\left(x_{k-1, j}, r\right)\right)$ $\cap\left\{y:\left|y-x_{k, i}\right|=v\right\}$. Let $g(v \mid u)$ be the length of that curve and its value is:

$$
\begin{aligned}
& g(v \mid u) \\
& = \begin{cases}2 v \operatorname{arcos} \frac{r^{2}-u^{2}-v^{2}}{2 u v} & \text { if } r-u<v<r ; \\
0 & \text { if } 0<v \leq r-u .\end{cases}
\end{aligned}
$$

Figure 1 demonstrates the branching process described here. Initially, the parent node $x_{k-1, j}$ and the child node $x_{k, i}$ with type $u$ are shown in Figure 1(a). The grandchildren nodes having type $v$ could occur along the circle centered at node $x_{k, i}$. The valid ones that contribute to percolation only appear along the curve from $A$ to $B$ with counter-clockwise direction in Figure 1(b). On the curve $A B$, a grandchildren nodes $x_{k+1, l}$. is placed. The equation (4) calculates the length of the curve $A B$.

Figure 1 Multi-type branching process: (a) the first branching and (b) the second branching (see online version for colours)

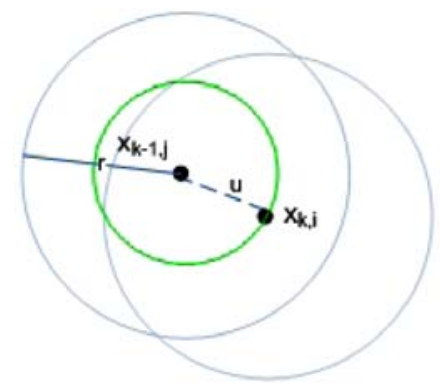

(a)

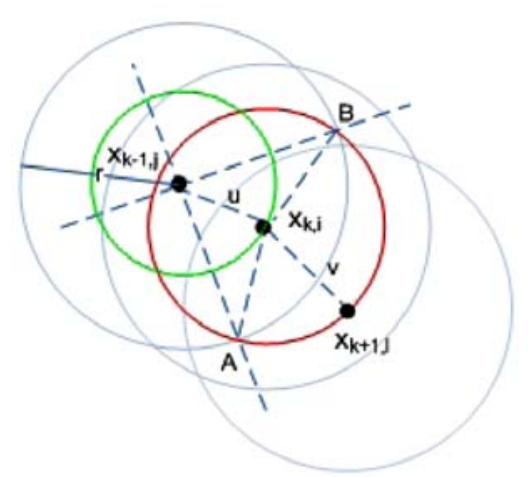

(b)
As a result, given that an individual is of type $u$, the expected total number of its children whose types lie in $(0, r)$ is given by

$$
\lambda \int_{0}^{r} g(v \mid u) d v
$$

From Theorem 3.10 in Meester and Roy (1996), the expected number of members of the $n$th generation having types in $(0, r)$ coming from a particular individual of type $u$ is given by

$$
\lambda^{n} \int_{0}^{r} g_{n}(v \mid u) d v
$$

Then, the expected total number of individuals is

$$
\sum_{n=1}^{\infty} \lambda^{n} \int_{0}^{r} g_{n}(v \mid u) d v
$$

By taking advantage of the linear operator $T_{f}$, the equation is further reduced to

$$
\sum_{n=1}^{\infty} \lambda^{n} T_{1}^{n}(u)
$$

where $\quad T_{f}(u)=\int_{0}^{r} f(v) g(v \mid u) d v \quad$ and $\quad 1(v) \equiv 1 \quad$ for $\quad$ all $v \in(0, r)$.

If $\lambda$ is sufficiently small, the result of branching process will converge. Specifically, $\lambda$ should be less than $\|T\|^{-1}$ where $\|T\|$ denotes the usual operator norm of $T$. Using the theory of Hilbert-Schmidt operator (Moiseiwitsch, 1977), $\|T\|$ is greater than $\frac{\int_{0}^{r} \int_{r-u}^{r} g(v \mid u) d v d u}{r}$, so the condition of convergence is

$$
\lambda<\frac{r}{\int_{0}^{r} \int_{r-u}^{r} g(v \mid u) d v d u} .
$$

The consequence of convergence demonstrates that the expected number of nodes in a component is finite, so the critical density $\lambda_{c}$ should be greater than the density with which the total number of nodes is convergent. Then the expression of lower bound of critical density is

$$
\lambda_{c}>\frac{r}{\int_{0}^{r} \int_{r-u}^{r} g(v \mid u) d v d u}
$$

\subsection{Upper bound of $\lambda_{c}$}

In order to obtain the upper bound of critical density, the site percolation model on the triangular lattice is applied and the process is based on the Theorem 3.10 in Meester and Roy (1996). Figure 2 shows a part of the triangular lattice. The vertex of each triangle is called site. It represents the area of the yellow 'flower' shown in that figure. The theory of site percolation (Kesten, 1982) says that any nodes located in the flower can be equal to the case that the incident vertex is occupied. So the condition of percolation on the triangular lattice is applied to the lattice covered by flowers centered at the vertex of triangle. 
Figure 2 The triangular lattice with area $A$ (see online version for colours)

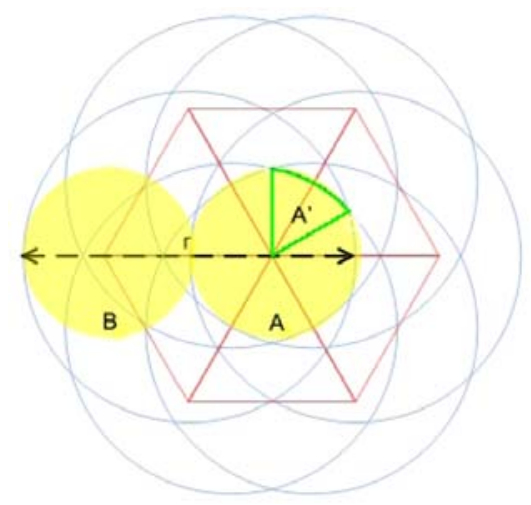

The rational is that if the site percolation of the triangular lattice occurs, the percolation occurs in the network. For the multihop network we study in this paper, a link occurs only if the distance between two nodes is within the transmission range $r$. Applying the site percolation model, we design the edge length of triangular lattice to be $\frac{r}{2}$. The sector forming the flower has a radius of $\frac{r}{2}$ and centers at the midpoints of the six edges adjacent to the same site.

An occupied site is defined if there is a node with Poisson Process $X$ situated inside the interior of the associated flower of the site. In Figure 2, that is the site $A$, representing the yellow flower. If there are two adjacent sites $A$ and $B$ both being occupied, the distances between the nodes centred at these two flowers must be less than or equal to twice the edge length of the triangular lattice. Since the edge length is $r / 2$, the largest distance between the two nodes in the adjacent flowers is $r$. Thus, the two nodes are linked consequently. Given that the lattice covers the entire network, we have proofed that the site percolation model described here allows the percolation to occur in the triangular lattice, hence in the network.

We use this model to derive the upper bound of the critical density. Assume the area of each site that has a flower shape be $|A|$ and the node density is $\lambda$. From Theorem 3.10 in Meester and Roy (1996), the probability of site occupation is $p=1-e^{-\lambda|A|}$. From the theory of site percolation (Kesten, 1982), if $p>1 / 2$ then with positive probability there is percolation in the site percolation model on the triangular lattice. Thus if $\lambda|A|>\ln 2$, then there is percolation with positive probability in the Boolean model.

To obtain $|A|$, we refer to Figure 2 , in that the area $A$ consists of six sectors of $A^{\prime}$. Each sector has a radius of $x$. It is easy to get the length of $x$, which is $\frac{r(\sqrt{15}-\sqrt{3}}{8}$. Then, the area $A$ is equal to $6 A^{\prime}$ ' which is approximately $0.2052 * r^{2}$. Thus the upper bound of the critical density is

$$
\lambda_{c}<\frac{\ln 2}{|A|} \approx \frac{3.3779}{r^{2}}
$$

\subsection{Discussion of $\lambda_{c}$}

As for the lower bound of critical density, the auxiliary function $a(r)$ is proposed to simplify the integral expression in equation (10). Then the non-zero part of $g(v \mid u)$ becomes $2 v a(r)$ where $a(r)=\operatorname{arcos} \frac{r^{2}-u^{2}-v^{2}}{2 u v}$ and the lower bound of critical density is reduced to $\frac{\frac{3}{2} a(r)}{r^{2}}$. It is difficult to derive the concrete form of $a(r)$, however its value range can be determined.

Lemma 4.1: Given $r$ which denotes the transmission range of node and $a(r)=\arccos \frac{r^{2}-u^{2}-v^{2}}{2 u v}$ where $0<u, v<r$ and $u+v>r$, then $a(r) \in\left(0, \frac{2 \pi}{3}\right)$.

Proof: Let $f(r, u, v)=\frac{r^{2}-u^{2}-v^{2}}{2 u v} \quad$ where $0<u, v<r$ and $u+v>r$, then $f_{u}^{\prime}=\frac{-r^{2}-u^{2}+v^{2}}{2 v u^{2}}<0$ and $f_{v}^{\prime}=$ $\frac{-r^{2}-v^{2}+u^{2}}{2 u v^{2}}<0$. So the extrema of function $f(r, u, v)$ can be found at the definition boundary. When $u+v=r$, $f(r, u, v)=1$. While $u, v=r, f(r, u, v)=-1 / 2$. Since $a(r)=\operatorname{arcos} \frac{r^{2}-u^{2}-v^{2}}{2 u v}$, then $a(r) \in\left(0, \frac{2 \pi}{3}\right)$.

According to the lower bound and upper bound of node density, the critical density falls in the following range: $\left(\frac{\frac{3}{2} a(r)}{r^{2}}, \frac{3.3779}{r^{2}}\right)$. Figure 3 shows the curves of the lower bound and the upper bound of the critical density with the transmission range $r$ increasing from 1-10. As seen from the figure, both curves decline when the transmission range increases. This explains the fact that when transmission range is large, a node can still maintain its connectivity while the nodes around it can be less. The figure also shows that when transmission range increases, the lower and the upper bounds become closer.

Figure 3 Upper bound and lower bound of $\lambda_{c}$ (see online version for colours)

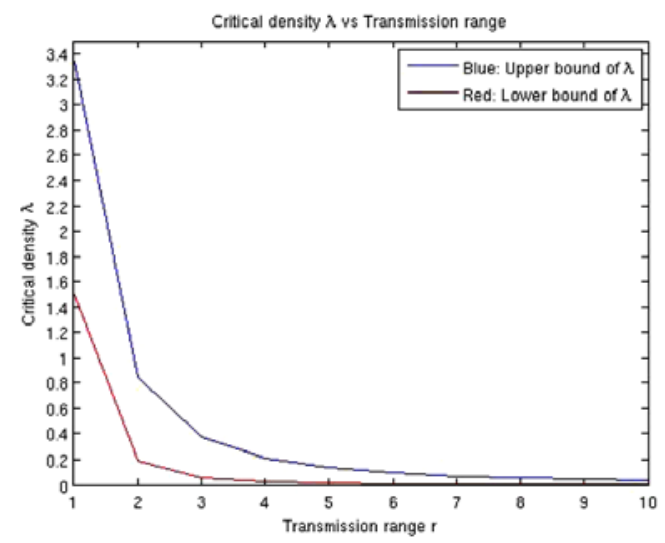




\subsection{Connectivity latency}

The time elapsed to reach the lower or upper bound depends on the growth function of the network density. We use three growth functions to derive the first connection time and total connection time according to the bounds we obtained previously. The three functions potentially depict different ways that nodes can be inserted to the network. The first function follows linear growth shown in the equation $x(t)=k t+a$ where $x(0)=a$. The coefficient $k$ denotes the growth rate and a can be viewed as the initial amount of nodes. Such a function reflects a constant rate increasing of nodes in the network, which can be true in some scenarios. The second is the exponential function to reflect the case that the growth of node happens in short time. The expression is $x(t)=a e^{k t}$ where $x(0)=a$. In this function, $k$ and $a$ represent the growth rate and initial number respectively. The last one is the logistic function which is often used to describe the population growth and virus propagation. Different from the other functions, the logistic function incorporates an upper bound on the growth. We denote the growth function by $x(t)=\frac{a}{1+e^{-k\left(t-t_{0}\right)}}$ where $x\left(t_{0}\right)=\frac{a}{2}$. The constant $a$ can be regarded as the maximum number of infected nodes, $k$ denotes the average infection rate and $t_{0}$ is the time when the growth rate reaches the maximum.

Three sample functions are illustrated in Figure 4 and the curves show the different growth patterns. The parameters for the three growth functions are selected in such a way that all of them will reach the maximum number of nodes 1400 by the simulation time of 90s. With this special constraint, the exponential function grows slower than linear at the beginning. It shows rapid growth rate toward the end of the simulation. The logistic function shows the trend of slower down when approaching the upper limit.

Figure 4 Growth functions (see online version for colours)

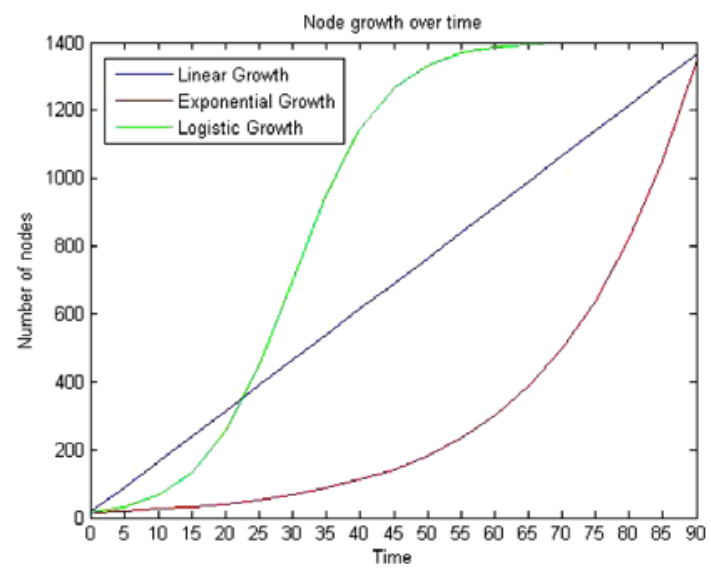

\section{Simulation}

We simulate the network evolution in Matlab with different growth functions to verify the bounds of critical density derived from the previous section. The simulation area is $20 \times 20$ with uniformly distributed nodes in two dimensional space. The network evolution starts from time 0 and time step is set to be $1 \mathrm{~s}$. The results are obtained from total 50 runs. The averaged values and confidence intervals with probability 0.9 are presented in many figures.

We measure the connectivity using Relative Giant Component Size. It is the ratio of the number of nodes in the largest network component to the total nodes. The largest network component can be found through Breadth First Search in the generated network. As the density of nodes increases, the ratio approaches to 1 .

Figures 5-7 describe the process of network evolution in terms of connection using different growth functions. The red circle denotes the node and blue line is the link between two nodes. As the network grows, more nodes and connections appear in the simulation area. In the linear growth function $x(t)=k t+a$ used in Figure 5, both parameter $k$ and $a$ are set to be 15 . For the exponential function $x(t)=a e^{k t}$ in Figure 6, we let the $a=15$ and $k=$ $1 / 20$ then the amount of node grows quickly next to the end of simulation. In Figure 7, the specific form of logistic growth function is $x(t)=\frac{1400}{1+e^{-0.15(t-30)}}$. The configuration of function shows that the most nodes will be generated around $30 \mathrm{~s}$.

Further, the relation between connectivity and time is illustrated. In Figure 8, the transmission range is set to be 1 . The red and green line in each subfigures (a)-(c) indicate the first connection time and the total connection time along with the corresponding densities. All the curves in Figure 8(a)-(c) roughly show the upward trend of relative size of giant network component. In the earlier phase, due to the smaller total number of nodes, the nodes in the largest component account for a relative high ratio. However, in the phase after the first connection time, the ratio shows an increasing mode and reaches to 1 when all the nodes are connected. In addition, the time instants of the first connection time and those of the total connection time in these subgraphs agree with the growth function each represents (see Figure 4). They also show that the corresponding transition periods, i.e., the length between the red line and green line, reflect the growth functions. As for the confidence interval with 0.9 probability, the intervals close to the lower bound denoted by green line have wider ranges. The observations reveal that the network state in terms of connectivity has larger variance during that period of time. The first connection time approximately captures the moment when the network connectivity begins to change. 
Figure 5 Network evolution with linear growth at time: (a) $t=0$; (b) $t=30$; (c) $t=60$ and (d) $t=90$ (see online version for colours)

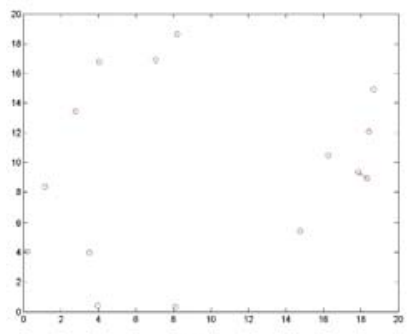

(a)

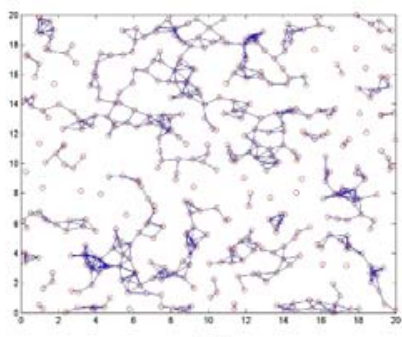

(b)

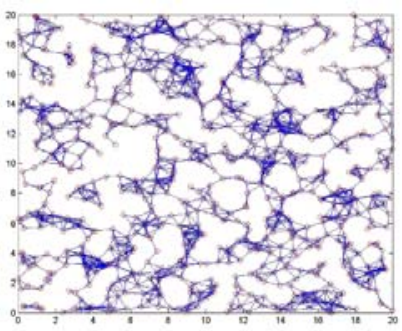

(c)

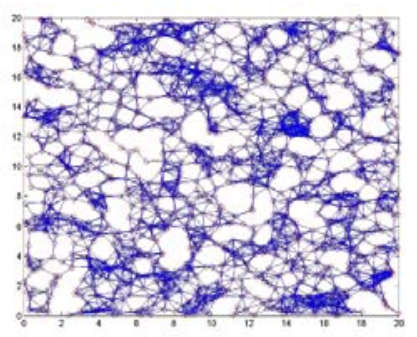

(d)

Figure 6 Network evolution with exponential growth at time: (a) $t=0$; (b) $t=30$; (c) $t=60$ and (d) $t=90$ (see online version for colours)

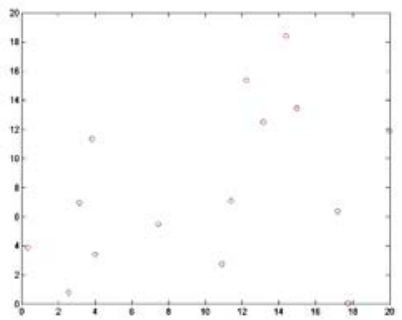

(a)

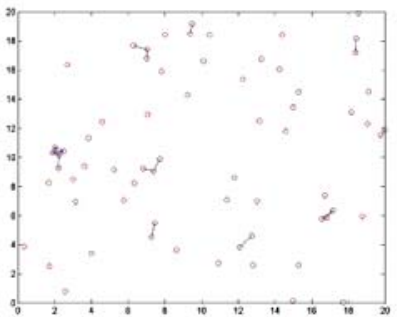

(b)

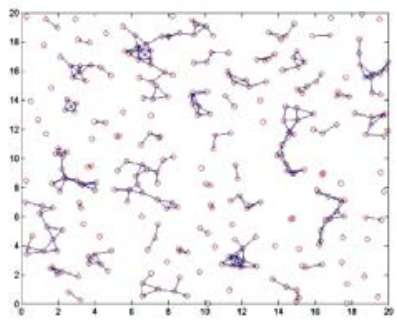

(c)

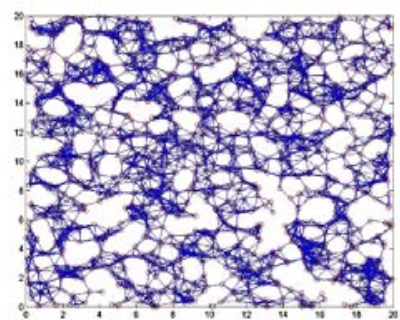

(d)

Figure 7 Network evolution with logistic growth at time: (a) $t=0$; (b) $t=30$; (c) $t=60$ and (d) $t=90$ (see online version for colours)

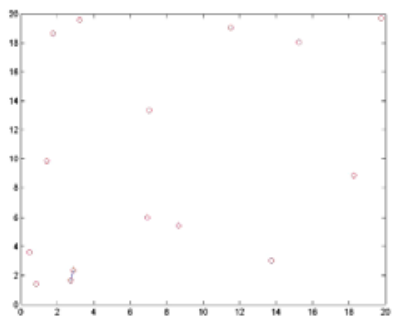

(a)

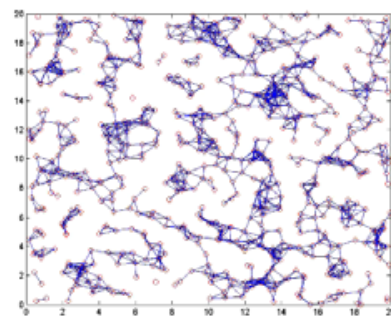

(b)

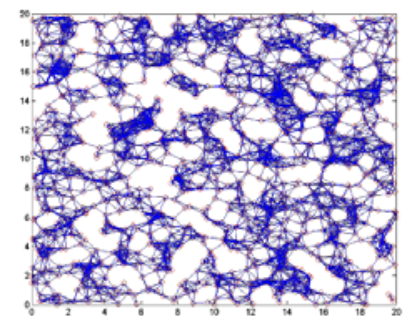

(c)

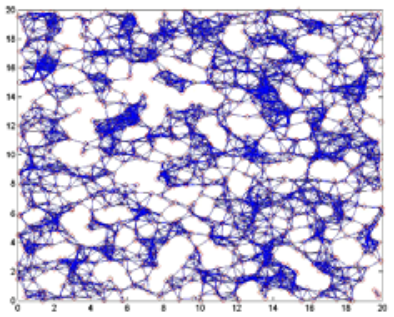

(d)

Figure 8 Phase transition against time with $r=1$ at time: (a) linear growth; (b) exponential growth and (c) logistic growth (see online version for colours)

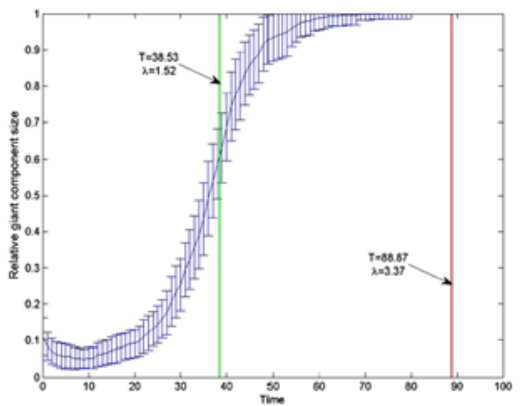

(a)

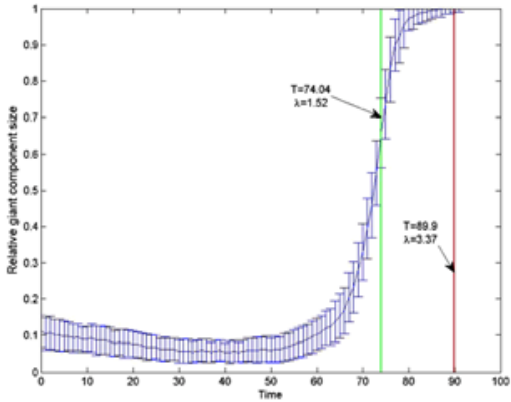

(b)

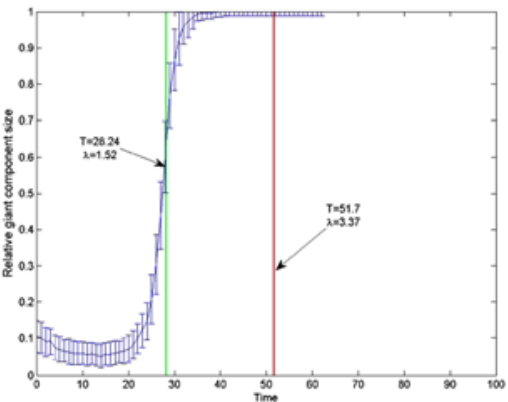

(c)
Figure 9 shows the expected number of node neighbours with short confidence intervals according to the three node growth functions. The curves in each subfigure approximate the growth functions in Figure 4. The expected number of neighbours corresponding to the lower bound of critical density is around four and the amount is about ten for the upper bound. The observation indicates that the network is connected when the amount of neighbour increases from four to ten. The connectivity metric in terms of expected neighbour conforms to the result of connection degree in Xue and Kumar (2004). Compared to the conventional knowledge of the magic number of node density, the lower bound we show here is less. This is because the conventional number serves more like an average case, while the lower bound here depicts the latest time for the network to have no probability of the occurrence of the giant component. It is not the time that the giant component will definitely occur. This also explains that the upper bound is larger than the conventional number. 
Figure 9 Expected number of neighbours against time with $r=1$ : (a) linear growth; (b) exponential growth and (c) logistic growth (see online version for colours)

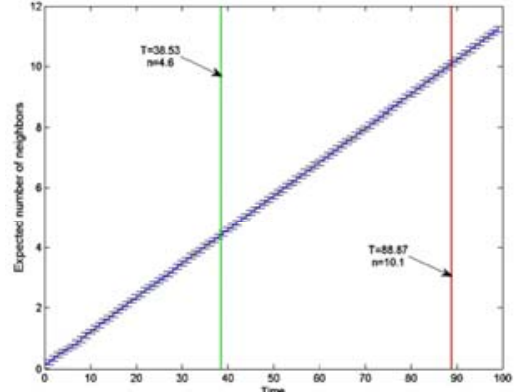

(a)

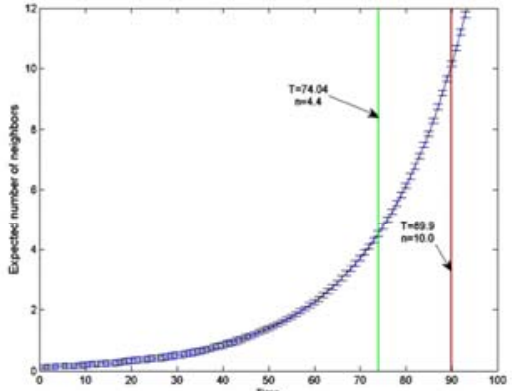

(b)

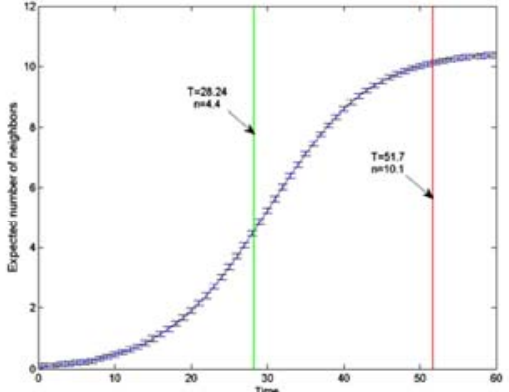

(c)

Figure 10 Phase transition against time with $r=2$ : (a) linear growth; (b) exponential growth and (c) logistic growth (see online version for colours)

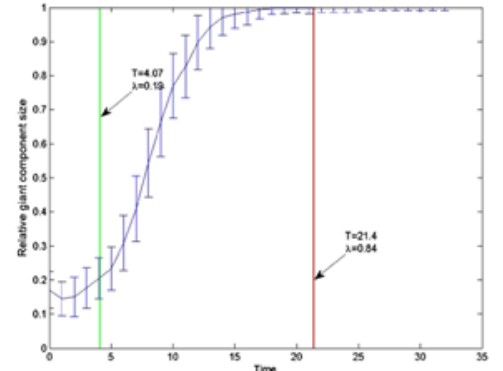

(a)

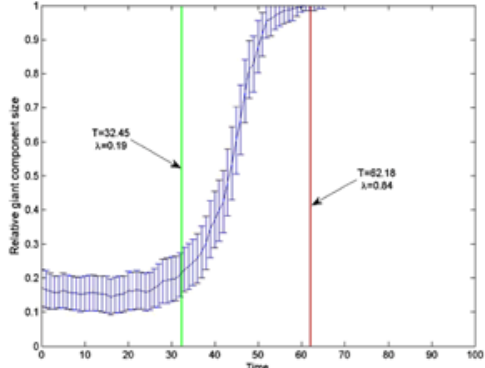

(b)

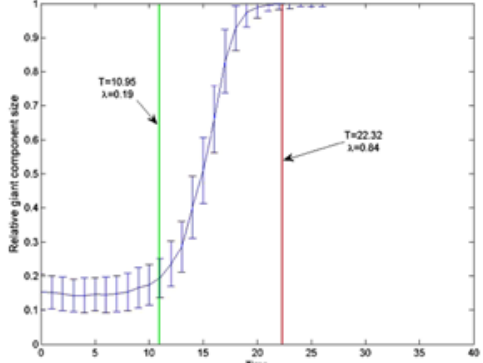

(c)

Figure 11 Expected number of neighbours against time with $r=2$ : (a) linear growth; (b) exponential growth and (c) logistic growth (see online version for colours)

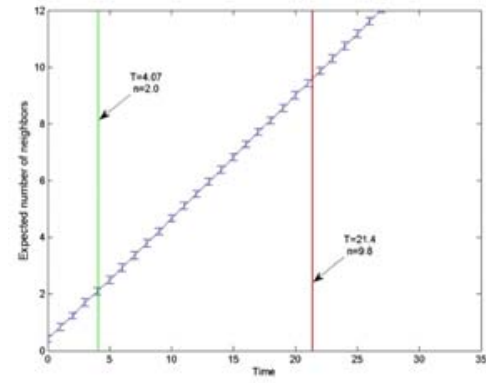

(a)

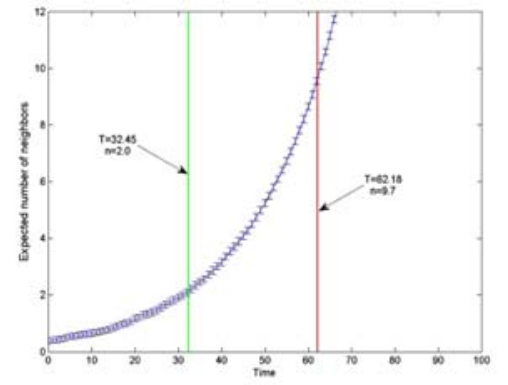

(b)

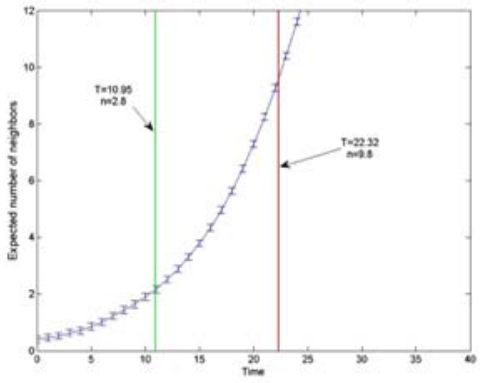

(c)
We then change the transmission range to be $r=2$ to study the connectivity transition. The growth functions are the same as above. The results are given in Figure 10. Compared with the curves for $r=1$ (Figure 8), these results show that less time is needed to reach the total connected state due to the larger $r$. Moreover, the first connection time occurs when the size of giant component is relative small. Figure 11 further shows the evolution of the expected neighbours in this $r=2$ case where the values approximately range from two to ten during the critical phase. Although the connection time becomes short due to the larger transmission range, the expected number of neighbour is relatively stable.

\section{Conclusion}

In the paper, we study the connectivity transition of wireless network when the density grows. The bounds of the related critical densities that denote the transformation from partition to connected state are derived, which are corresponding to the first connection time and total connection time. We studied three node growth functions for modeling the increment of node. The simulations use three instances of these growth functions and the results show that the related time metrics and the trend of change in connectivity when time goes by. In future work, parameters can be studied for more realistic radio models in which the transmission range could have irregular shapes rather than a circle. Also the heterogeneity of wireless nodes can be considered such as different transmission ranges and heterogeneous distribution in deployments.

\section{Acknowledgement}

This work is supported in part by NSF Awards No. 0829827. 


\section{References}

Bettstetter, C. (2002) 'On the minimum node degree and connectivity of a wireless multihop network', Proceedings of the 3rd ACM International Symposium on Mobile ad hoc Networking and Computing, Lausanne, Switzerland.

Bollobas, B. and Riordan, O. (2006) Percolation, Cambridge University Press, Cambridge, UK.

Chen, Y., Borrel, V., Ammar, M. and Zegura, E. (2011) 'A framework for characterising the wireless and mobile network continuum', ACM SIGCOMM Compute Communication Review, Vol. 41, No. 1, pp.5-13.

Dousse, O., Mannersalo, P. and Thiran, P. (2004) 'Latency of wireless sensor networks with uncoordinated power saving mechanisms', Proceedings of the 5th ACM International Symposium on Mobile ad hoc Networking and Computing, Roppongi Hills, Tokyo, Japan, pp.109-120.

Dousse, O., Baccelli, F. and Thiran, P. (2005) 'Impact of interferences on connectivity in ad hoc networks', IEEE/ACM Transactions on Networking (TON), Vol. 13, No. 2, pp.425-436.

Grimmett, G.R. and Stirzaker, D.R. (1992) Probability and Random Processes, Clarendon Press, Oxford.

Gupta, P. and Kumar, P.R. (1998) 'Critical power for asymptotic connectivity in wireless networks', in McEneany, W.M., Yin, G. and Zhang, Q. (Eds): Stochastic Analysis, Control, Optimisation and Applications, A Volume in Honor of W.H. Fleming, pp.547-566.

Kesten, H. (1982) Percolation Theory for Mathematics, Birkhäuser, Boston.
Kong, Z. and Yeh, E.M. (2007) 'Distributed energy management algorithm for large-scale wireless sensor networks', Proceedings of the 8th ACM International Symposium on Mobile Ad Hoc Networking and Computing, Montreal, Quebec, Canada, pp.209-218.

Liggett, T. (1985) 'An improved subadditive ergodic theorem', The Annals of Probability, Vol. 13, No. 4, pp.1279-1285.

Meester, R. and Roy, R. (1996) Continuum Percolation, Cambridge University Press, Cambridge, England. Moiseiwitsch, B.L. (1977) Integral Equations, Longman, Prentice Hall, Press, New Jersey.

Penrose, M. (2006) Random Geometric Graphs, Oxford University Press, New York. Ramanathan, R. and Rosales-hain, R. (2000) 'Topology control of multihop wireless networks using transmit power adjustment', IEEE INFOCOM, March, Tel Aviv, Israel, pp.404-413.

Sanchez, M., Manzoni, P. and Haas, Z.J. (1999) 'Determination of critical transmission range in ad-hoc networks', Multiaccess, Mobility and Teletraffic for Wireless Communications, October, Venice, Italy, pp.293-303.

Xing, F. and Wang, W. (2008) 'On the critical phase transition time of wireless multi-hop networks with random failures', Proceedings of the 14th ACM International Conference on Mobile Computing and Networking, San Francisco, California, USA.

Xue, F. and Kumar, P. (2004) 'The number of neighbors needed for connectivity of wireless networks', Kluwer Wireless Networks, Vol. 10, No. 2, pp.169-181. 